\title{
KINEMATIC SYNTHESIS OF SPATIAL LINKAGES WITH SPHERICAL PAIRS
}

\author{
Serikbay Kosbolov, Algazy Zhauyt, Serikbol Kosbolov \\ Kazakh National Technical University named after K.I. Satpayev, Department of Applied Mechanics and Basics of Machine \\ Design, Almaty, Kazakhstan; e-mail: kosbolov@mail.ru; ali84jauit@mail.ru; serikbol_k@mail.ru
}

\begin{abstract}
A solution to the problem of synthesizing an initial three-dimensional kinematic chain with spherical and rotary kinematic pairs is presented. It is shown that this chain can be used as a structural module for structural-kinematic synthesis of motion of a three-dimensional four-link generating lever mechanisms by preset positions of the input and output links.
\end{abstract}

Keywords: mechanism, four-link, kinematic pairs, kinematic chains, synthesis

\section{Introduction}

Some papers demonstrate that four-link basic kinematic chains (BKC) may be used as a structural module with structural and kinematic synthesis of plain linkage mechanisms. Such an approach to the synthesis of plain mechanisms allows reducing the problem of their structural and kinematic synthesis to solution of the problem of BKC synthesis (Joldasbekov et al., 1987), which is very useful for automation of mechanisms engineering. This paper testifies that a specified approach may be applied to the problem of structural and kinematic synthesis of spatial linkage mechanisms (Kosbolov and Rakhmatulina, 2012b). The solution of the problem of synthesis of spatial BKC of RSS type (R - rotational, S - spherical kinematic pairs) is represented, and its use as a structural module with structural and kinematic synthesis of spatial linkage mechanisms through predetermined positions of input and output links is shown (Kosbolov et al., 2005). A method of solving the problem of BKC synthesis of RSS type is based on the introduction of two movable bodies invariably associated with the input and output links (Kosbolov and Rakhmatulina, 2013b).

\section{On the existence of solution to the problem of initial kinematic chain synthesis with spherical kinematic pairs}

Problem statement: given $N$ of finite distant positions of two solids $Q_{1}$ and $Q_{2}$

$$
Q_{1}\left(\theta_{i}^{1}, \psi_{i}^{1}, \phi_{i}^{1}\right) \quad Q_{2}\left(X_{D i}, Y_{D i}, Z_{D i}, \theta_{i}^{2}, \psi_{i}^{2}, \phi_{i}^{2}\right) \quad i=\overline{1, N}
$$

where $\theta_{i}^{j}, \psi_{i}^{j}, \phi_{i}^{j}$ are fixed axis Eulerian angles $O X Y Z$ and $X_{D i}, Y_{D i}, Z_{D i}$ are coordinates of the point $D_{i}$ of the solid $Q_{2}$.

It is required to find such points in the fixed axis as $A\left(X_{A}, Y_{A}, Z_{A}\right)$, of the solid $Q_{1}$ and $C\left(x_{C}, y_{C}, z_{C}\right)$ of the solid $Q_{2}$, so that distance between the points $B$ and $C$ in all positions of the solids $Q_{1}$ and $Q_{2}$ is little different from some constant value $R$ (Fig. 1).

Problem solution: Let us introduce a weighted difference for the $i$-th position of the solids in form

$$
\Delta_{q_{i}}=\left|\overrightarrow{B_{i} C_{i}}\right|^{2}-R^{2}=\left(X_{C_{i}}-X_{B_{i}}\right)^{2}+\left(Y_{C_{i}}-Y_{B_{i}}\right)^{2}+\left(Z_{C_{i}}-Z_{B_{i}}\right)^{2}-R^{2} \quad i=\overrightarrow{1, N}
$$




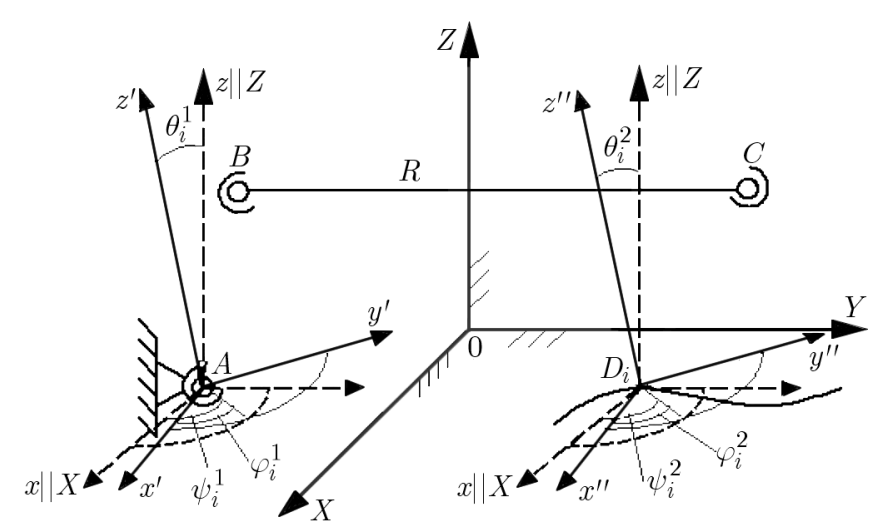

Fig. 1. Equivalent four-link kinematic chain $A B C D$

where

$$
\begin{aligned}
& {\left[\begin{array}{c}
X_{B_{i}} \\
Y_{B_{i}} \\
Z_{B_{i}} \\
1
\end{array}\right]=\left[\begin{array}{cccc} 
& & X_{A} \\
& \mathbf{T}_{10}^{i} & Y_{A} \\
0 & 0 & 0 & Z_{A}
\end{array}\right]\left[\begin{array}{c}
x_{B} \\
y_{B} \\
z_{B} \\
1
\end{array}\right]} \\
& {\left[\begin{array}{c}
X_{C_{i}} \\
Y_{C_{i}} \\
Z_{C_{i}} \\
1
\end{array}\right]=\left[\begin{array}{cccc}
\mathbf{T}_{20}^{i} & & Y_{D i} \\
0 & 0 & 0 & 1
\end{array}\right]\left[\begin{array}{c}
x_{C} \\
y_{C} \\
z_{C} \\
1
\end{array}\right]}
\end{aligned}
$$

and

$$
\mathbf{T}_{j 0}^{i}=\left[\begin{array}{ccc}
e_{i 1}^{\prime} & e_{i 2}^{\prime} & e_{i c}^{\prime} \\
m_{i 1}^{\prime} & m_{i 2}^{\prime} & m_{i c}^{\prime} \\
n_{i 1}^{\prime} & n_{i 2}^{\prime} & n_{i c}^{\prime}
\end{array}\right] \quad \begin{aligned}
& j=\overline{1,2} \\
& \hline=\overline{1, N}
\end{aligned}
$$

where

$$
\begin{aligned}
& e_{i 1}^{j}=\cos \psi_{i}^{j} \cos \phi_{i}^{j}-\cos \theta_{i}^{j} \sin \psi_{i}^{j} \sin \phi_{i}^{j} \\
& m_{i 1}=\sin \psi_{i}^{j} \cos \phi_{i}^{j}+\cos \theta_{i}^{j} \cos \psi_{i}^{j} \cos \phi_{i}^{j} \\
& n_{i 1}^{j}=\sin \theta_{i}^{j} \sin \phi_{i}^{j} \\
& e_{i 2}^{j}=-\cos \psi_{i}^{j} \sin \phi_{i}^{j}-\cos \theta_{i}^{j} \sin \psi_{i}^{j} \cos \phi_{i}^{j} \\
& m_{i 2}^{j}=-\sin \psi_{i}^{j} \sin \phi_{i}^{j}+\cos \theta_{i}^{j} \cos \psi_{i}^{j} \sin \phi_{i}^{j} \\
& n_{i 2}^{j}=\sin \theta_{i}^{j} \cos \phi_{i}^{j} \\
& e_{i 3}^{j}=\sin \theta_{i}^{j} \sin \psi_{i}^{j} \\
& m_{i 3}^{j}=-\sin \theta_{i}^{j} \cos \psi_{i}^{j} \\
& n_{i 3}^{j}=\cos \phi_{i}^{j}
\end{aligned}
$$

It is a function of ten parameters: $X_{A}, Y_{A}, Z_{A}, x_{B}, y_{B}, z_{B}, R, x_{C}, y_{C}, z_{C}$. By grouping these parameters in fours with the common parameter $R$, let us represent the weighted difference in three different forms (McCarthy, 1995; Golynski, 1970; Innocenti, 1995; Kosbolov and Rakhmatulina, 2012b, 2013b,c; Kosbolov et al., 2014)

$$
\begin{aligned}
\Delta_{q_{i}}^{(1)} & =\left(\widetilde{X}_{A_{i}}-X_{A}\right)^{2}+\left(\widetilde{Y}_{A_{i}}-Y_{A}\right)^{2}+\left(\widetilde{Z}_{A_{i}}-Z_{A}\right)^{2}-R^{2} \\
\Delta_{q_{i}}^{(2)} & =\left(\widetilde{x}_{B_{i}}-x_{B}\right)^{2}+\left(\widetilde{y}_{B_{i}}-y_{B}\right)^{2}+\left(\widetilde{z}_{B_{i}}-Z_{B}\right)^{2}-R^{2} \\
\Delta_{q_{i}}^{(3)} & =\left(\widetilde{x}_{C_{i}}-x_{C}\right)^{2}+\left(\widetilde{y}_{C_{i}}-y_{C}\right)^{2}+\left(\widetilde{z}_{C_{i}}-Z_{C}\right)^{2}-R^{2}
\end{aligned}
$$


where

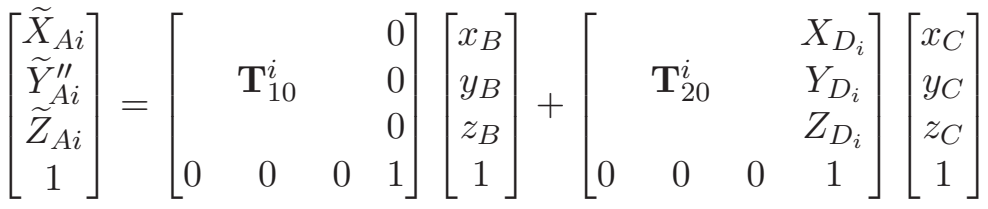

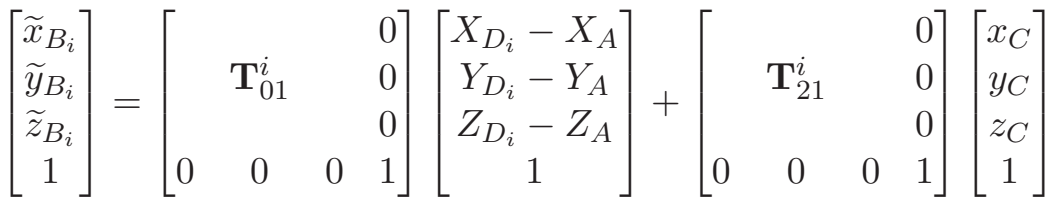

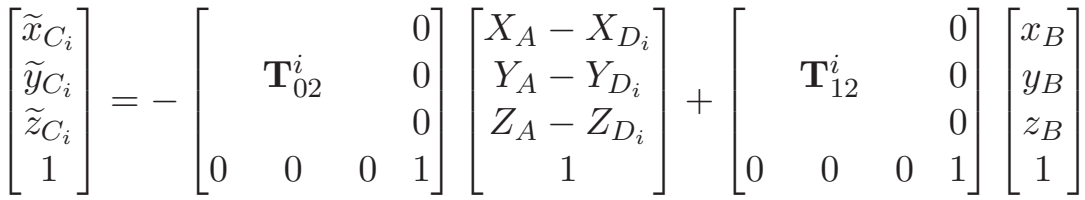

where $\mathbf{T}_{k j}^{i}$ is the transfer matrix from the $k$ coordinate system to the $j$ system determined as

$$
\mathbf{T}_{01}^{i}=\left[\mathbf{T}_{10}^{i}\right]^{\mathrm{T}} \quad \mathbf{T}_{02}^{i}=\left[\mathbf{T}_{20}^{i}\right]^{\mathrm{T}} \quad \mathbf{T}_{21}^{i}=\mathbf{T}_{01}^{i} \times \mathbf{T}_{20}^{i} \quad \mathbf{T}_{12}^{i}=\mathbf{T}_{02}^{i} \times \mathbf{T}_{10}^{i}
$$

The necessary conditions for minimum of the sum of squares of the weighted difference

$$
\left.S=\sum_{i=1}^{N}\left[\Delta_{q_{i}}^{(k)}\right]^{2} \quad k=1,2,3\right)
$$

may be written as the following system of equations

$$
\begin{array}{llll}
\frac{\partial S}{\partial X_{A}}=0 & \frac{\partial S}{\partial Y_{A}}=0 & \frac{\partial S}{\partial Z_{A}}=0 & \frac{\partial S}{\partial R}=0 \\
\frac{\partial S}{\partial x_{B}}=0 & \frac{\partial S}{\partial y_{B}}=0 & \frac{\partial S}{\partial z_{B}}=0 & \frac{\partial S}{\partial R}=0 \\
\frac{\partial S}{\partial x_{C}}=0 & \frac{\partial S}{\partial y_{C}}=0 & \frac{\partial S}{\partial z_{C}}=0 & \frac{\partial S}{\partial R}=0
\end{array}
$$

From $(2.10)_{1}$, considering $(2.6)_{1}$ and $(2.9)$, we obtain

$$
\begin{array}{lll}
\sum_{i=1}^{N} \Delta_{q_{i}}^{(1)}\left(\widetilde{X}_{A_{i}}-X_{A}\right)=0 & & \sum_{i=1}^{N} \Delta_{q_{i}}^{(1)}\left(\widetilde{Y}_{A_{i}}-Y_{A}\right)=0 \\
\sum_{i=1}^{N} \Delta_{q_{i}}^{(1)}\left(\widetilde{Z}_{A_{i}}-Z_{A}\right)=0 & & \sum_{i=1}^{N} \Delta_{q_{i}}^{(1)} R=0
\end{array}
$$

Assume that $R \neq 0$. Then from the last equality of system (2.11), it follows that

$$
\sum_{i=1}^{N} \Delta_{q_{i}}^{(1)}=0
$$

With provision for (2.12), the system of equations (2.11) takes the form

$$
\sum_{i=1}^{N} \Delta_{q_{i}}^{(1)} \widetilde{X}_{A i}=0 \quad \sum_{i=1}^{N} \Delta_{q_{i}}^{(1)} \widetilde{Y}_{A i}=0 \quad \sum_{i=1}^{N} \Delta_{q_{i}}^{(1)} \widetilde{Z}_{A i}=0 \quad \sum_{i=1}^{N} \Delta_{q_{i}}^{(1)}=0
$$


By substituting expressions for $\Delta_{q_{i}}^{(1)}$ from $(2.6)_{1}$ into system (2.13), we obtain

$$
\begin{aligned}
& \sum_{i=1}^{N}\left[\widetilde{X}_{A i}^{2} X_{A}+\widetilde{X}_{A i} \widetilde{Y}_{A i} Y_{A}+\widetilde{Z}_{A i} \tilde{X}_{A i} Z_{A}+\frac{1}{2}\left(R^{2}-X_{A}^{2}-Y_{A}^{2}-Z_{A}^{2}\right) \widetilde{X}_{A i}\right] \\
& =\frac{1}{2} \sum_{i=1}^{N}\left(\widetilde{X}_{A i}^{2}+\widetilde{Y}_{A i}^{2}+\widetilde{Z}_{A i}^{2}\right) \widetilde{X}_{A i} \\
& \sum_{i=1}^{N}\left[\widetilde{X}_{A i} \widetilde{Y}_{A i} X_{A}+\widetilde{Y}_{A i}^{2} Y_{A}+\widetilde{Z}_{A i} \widetilde{Y}_{A i} Z_{A}+\frac{1}{2}\left(R^{2}-X_{A}^{2}-Y_{A}^{2}-Z_{A}^{2}\right) \widetilde{Y}_{A i}\right] \\
& =\frac{1}{2} \sum_{i=1}^{N}\left(\widetilde{X}_{A i}^{2}+Y_{A i}^{2}+Z_{A i}^{2}\right) \widetilde{Y}_{A i} \\
& \sum_{i=1}^{N}\left[\widetilde{Z}_{A i} \widetilde{X}_{A i} X_{A}+\widetilde{Y}_{A i} \widetilde{Z}_{A i} Y_{A}+\widetilde{Z}_{A i}^{2} Z_{A}+\frac{1}{2}\left(R^{2}-X_{A}^{2}-Y_{A}^{2}-Z_{A}^{2}\right) \widetilde{Z}_{A i}\right] \\
& =\frac{1}{2} \sum_{i=1}^{N}\left(\widetilde{X}_{A i}^{2}+\widetilde{Y}_{A i}^{2}+\widetilde{Z}_{A i}^{2}\right) \widetilde{Z}_{A i} \\
& \sum_{i=1}^{N}\left[\widetilde{X}_{A i} X_{A}+\widetilde{Y}_{A i} Y_{A}+\widetilde{Z}_{A i} Z_{A}+\frac{1}{2}\left(R^{2}-X_{A}^{2}-Y_{A}^{2}-Z_{A}^{2}\right) \widetilde{X}_{A i}\right]=\frac{1}{2} \sum_{i=1}^{N}\left(\widetilde{X}_{A i}^{2}+\widetilde{Y}_{A i}^{2}+\widetilde{Z}_{A i}^{2}\right)
\end{aligned}
$$

System (2.14) is linear with respect to the variables $X_{A}, Y_{A}, Z_{A}$ and $H_{1}=\left(R^{2}-X_{A}^{2}-Y_{A}^{2}-\right.$ $\left.Z_{A}^{2}\right) / 2$, thus it may be written as

$$
\left[\begin{array}{cccc}
\sum_{i=1}^{N} \widetilde{X}_{A i}^{2} & \sum_{i=1}^{N} \widetilde{X}_{A i} \widetilde{Y}_{A i} & \sum_{i=1}^{N} \widetilde{X}_{A i} \widetilde{Z}_{A i} & \sum_{i=1}^{N} \widetilde{X}_{A i} \\
\sum_{i=1}^{N} \widetilde{X}_{A i} \widetilde{Y}_{A i} & \sum_{i=1}^{N} \widetilde{Y}_{A i} & \sum_{i=1}^{N} \widetilde{Y}_{A i} \widetilde{Z}_{A i} & \sum_{i=1}^{N} \widetilde{Y}_{A i} \\
\sum_{i=1}^{N} \widetilde{X}_{A i} \widetilde{Z}_{A i} & \sum_{i=1}^{N} \widetilde{Y}_{A i} \widetilde{Z}_{A i} & \sum_{i=1}^{N} \widetilde{Z}_{A i}^{2} & \sum_{i=1}^{N} \widetilde{Z}_{A i} \\
\sum_{i=1}^{N} \widetilde{X}_{A i} & \sum_{i=1}^{N} \widetilde{Y}_{A i} & \sum_{i=1}^{N} \widetilde{Z}_{A i} & N
\end{array}\right]\left[\begin{array}{c}
X_{A} \\
Y_{A} \\
Z_{A} \\
H_{1}
\end{array}\right]=\frac{1}{2}\left[\begin{array}{c}
\sum_{i=1}^{N} R_{A_{i}}^{2} \widetilde{X}_{A_{i}} \\
\sum_{i=1}^{N} R_{A_{i}}^{2} \widetilde{Y}_{A_{i}} \\
\sum_{i=1}^{N} R_{A_{i}}^{2} \widetilde{Z}_{A_{i}} \\
\sum_{i=1}^{N} R_{A_{i}}^{2}
\end{array}\right]
$$

where $R_{A i}^{2}=\tilde{X}_{A i}^{2}+\tilde{Y}_{A i}^{2}+\widetilde{Z}_{A i}^{2}$.

The solution to this system by Cramer's rule is as follows

$$
\left(X_{A}, Y_{A}, Z_{A}, H_{1}\right)=\frac{1}{D_{1}}\left(D_{X_{A}}, D_{Y_{A}}, D_{Z_{A}}, D_{H_{1}}\right) \quad D_{1} \neq 0
$$

Similarly, from $(2.10)_{2}$, considering $(2.6)_{2}$ and (2.9), we obtain a system of linear equations in the unknowns $x_{B}, y_{B}, z_{B}, H_{2}$

$$
\left[\begin{array}{cccc}
\sum_{i-1}^{N} \widetilde{x}_{B i}^{2} & \sum_{i-1}^{N} \widetilde{x}_{B i} y_{B i} & \sum_{i-1}^{N} \widetilde{x}_{B i} \widetilde{z}_{B i} & \sum_{i-1}^{N} \widetilde{x}_{B i} \\
\sum_{i-1}^{N} \widetilde{x}_{B i} y_{B i} & \sum_{i-1}^{N} \widetilde{y}_{B i}^{2} & \sum_{i-1}^{N} \widetilde{y}_{B i} \widetilde{z}_{B i} & \sum_{i-1}^{N} \widetilde{y}_{B i} \\
\sum_{i-1}^{N} \widetilde{x}_{B i} \widetilde{z}_{B i} & \sum_{i-1}^{N} \widetilde{y}_{B i} \widetilde{z}_{B i} & \sum_{i-1}^{N} \widetilde{z}_{B i}^{2} & \sum_{i-1}^{N} \widetilde{z}_{B i} \\
\sum_{i-1}^{N} \widetilde{x}_{B i} & \sum_{i-1}^{N} \widetilde{y}_{B i} & \sum_{i-1}^{N} \widetilde{z}_{B i} & N
\end{array}\right]\left[\begin{array}{c}
x_{B} \\
y_{B} \\
z_{B} \\
H_{2}
\end{array}\right]=\frac{1}{2}\left[\begin{array}{c}
\sum_{i=1}^{N} R_{B i}^{2} \widetilde{x}_{B i} \\
\sum_{i=1}^{N} R_{B i}^{2} \widetilde{y}_{B i} \\
\sum_{i=1}^{N} R_{B i}^{2} \widetilde{z}_{B i} \\
\sum_{i=1}^{N} R_{B i}^{2}
\end{array}\right]
$$

By solving this system by Cramer's rule, we obtain

$$
\left(x_{B}, y_{B}, z_{B}, H_{2}\right)=\frac{1}{D_{2}}\left(D_{x_{B}}, D_{y_{B}}, D_{z_{B}}, D_{H_{2}}\right) \quad D_{2} \neq 0
$$


From $(2.10)_{3}$, considering $(2.6)_{3}$ and $(2.10)_{1}$, we obtain a system of linear equations in the unknowns $x_{C}, y_{C}, z_{C}, H_{3}$

$$
\left[\begin{array}{cccc}
\sum_{i-1}^{N} \widetilde{x}_{C i}^{2} & \sum_{i-1}^{N} \widetilde{x}_{C i} \widetilde{y}_{C i} & \sum_{i-1}^{N} \widetilde{x}_{C i} \widetilde{z}_{C i} & \sum_{i-1}^{N} \widetilde{x}_{C i} \\
\sum_{i-1}^{N} \widetilde{x}_{C i} \widetilde{y}_{C i} & \sum_{i-1}^{N} \widetilde{y}_{C i}^{2} & \sum_{i-1}^{N} \widetilde{y}_{C i} \widetilde{z}_{C i} & \sum_{i-1}^{N} \widetilde{y}_{C i} \\
\sum_{i-1}^{N} \widetilde{x}_{C i} \widetilde{z}_{C i} & \sum_{i-1}^{N} \widetilde{y}_{C i} \widetilde{z}_{C i} & \sum_{i-1}^{N} \widetilde{z}_{C i}^{2} & \sum_{i-1}^{N} \widetilde{z}_{C i} \\
\sum_{i-1}^{N} \widetilde{x}_{C i} & \sum_{i-1}^{N} \widetilde{y}_{C i} & \sum_{i-1}^{N} \widetilde{z}_{C i} & N
\end{array}\right]\left[\begin{array}{c}
x_{C} \\
y_{C} \\
z_{C} \\
H_{3}
\end{array}\right]=\frac{1}{2}\left[\begin{array}{c}
\sum_{i=1}^{N} R_{C i}^{2} \widetilde{x}_{C i} \\
\sum_{i=1}^{N} R_{C i}^{2} \widetilde{y}_{C i} \\
\sum_{i=1}^{N} R_{C i}^{2} \widetilde{z}_{C i} \\
\sum_{i=1}^{N} R_{C i}^{2}
\end{array}\right]
$$

From which we obtain $x_{C}, y_{C}, z_{C}, H_{3}$

$$
\left(x_{C}, y_{C}, z_{C}, H_{3}\right)=\frac{1}{D_{3}}\left(D_{x_{C}}, D_{y_{C}}, D_{z_{C}}, D_{H_{3}}\right) \quad D_{3} \neq 0
$$

Eliminating the first four unknowns $X_{A}, Y_{A}, Z_{A}, R$, based on formula (2.15), it is possible to bring system (2.10) to a system of six equations with six unknowns $x_{B}, y_{B}, z_{B}, x_{C}, y_{C}, z_{C}$, which is convenient to be given as

$$
\begin{array}{lll}
\sum_{i=1}^{N} \Delta_{q_{i}}^{(1)} \frac{\partial \Delta_{q_{i}}^{(2)}}{\partial x_{B}}=0 & \sum_{i=1}^{N} \Delta_{q_{i}}^{(1)} \frac{\partial \Delta_{i}^{(3)}}{\partial x_{C}}=0 \\
\sum_{i=1}^{N} \Delta_{q_{i}}^{(1)} \frac{\partial \Delta_{q_{i}}^{(2)}}{\partial y_{B}}=0 & \sum_{i=1}^{N} \Delta_{q_{i}}^{(1)} \frac{\partial \Delta_{i}^{(3)}}{\partial y_{C}}=0 \\
\sum_{i=1}^{N} \Delta_{q_{i}}^{(1)} \frac{\partial \Delta_{q_{i}}^{(2)}}{\partial z_{B}}=0 & \sum_{i=1}^{N} \Delta_{q_{i}}^{(1)} \frac{\partial \Delta_{i}^{(3)}}{\partial z_{C}}=0
\end{array}
$$

Apparently, equations of this system are the same as the three equations of the thirteen degree in the three unknown functions given in the work by Kosbolov et al. (2005), though in this case we have a system of six equations in six unknown functions. Solution of system (2.21) is labor-intensive task, so it is more effective to apply a search algorithm for the minimum of the function $S$ stated below:

1. Give arbitrarily reference points $B^{(0)} \in Q_{1}, C^{(0)} \in Q_{2}$.

2. Solve the system of linear equations (2.16) and determine $X_{A}^{(1)}, Y_{A}^{(1)}, Z_{A}^{(1)}, R_{1}^{(1)}$.

3. Give points $A^{(1)} \in Q, C^{(0)} \in Q_{2}$.

4. Solve the system of equations (2.18) and determine $x_{B}^{(1)}, y_{B}^{(1)}, z_{B}^{(1)}, R_{2}^{(1)}$.

5. Give points $A^{(1)} \in Q, B^{(1)} \in Q_{1}$.

6. Solve the system of equations $(2.20)$ and determine $x_{C}^{(1)}, y_{C}^{(1)}, z_{C}^{(1)}, R_{3}^{(1)}$.

7. Check $\left|X_{A}^{i+1}-X_{A}^{i}\right| \leqslant \varepsilon,\left|Y_{A}^{i+1}-Y_{A}^{i}\right| \leqslant \varepsilon,\left|Z_{A}^{i+1}-Z_{A}^{i}\right| \leqslant \varepsilon,\left|R^{i+1}-R^{i}\right| \leqslant \varepsilon$.

8. If this condition is satisfied, the iterating is completed.

9. If this condition is not satisfied, proceed to item 1 by replacing the reference points $B^{(0)}$ and $C^{(0)}$ for the found points $B^{(1)}$ and $C^{(0)}$.

10. Then check the accuracy of the prescribed function reproduction by analysis of the position RKC $A B C D$

$$
\mathbf{r}_{D_{0}}=\mathbf{T}_{10} \mathbf{T}_{21} \mathbf{T}_{32} \mathbf{r}_{D_{3}}
$$


11. The iterating is completed, if the accuracy of reproduction satisfies the prescribed function. If it does not satisfy the prescribed accuracy, it is necessary to proceed to item 1 of the given algorithm.

By applying the algorithm, we obtain a decreasing sequence of values of the objective function $S_{1}^{(1)}, S_{2}^{(1)}, S_{3}^{(1)}, S_{1}^{(2)}, S_{2}^{(2)}, S_{3}^{(2)}$ which has a limit equal to the value of the function $S$ at the point of local minimum. When satisfying the inequality

$$
\max \left(\left|R^{(i)}-R^{(i-1)}\right|,\left|X_{A}^{(i)}-X_{A}^{(i-1)}\right|,\left|Y_{A}^{(i)}-Y_{A}^{(i-1)}\right|,\left|Z_{A}^{(i)}-Z_{A}^{(i-1)}\right|\right) \leqslant \varepsilon
$$

where $\varepsilon$ is the prescribed calculation accuracy, the iterating is completed. Convergence of the suggested algorithm is proved by the Weierstrass theorem.

Weierstrass theorem: For each function $f(x)$ continuous over $[a, b]$ and any real number $\varepsilon>0$, such a polynomial $p(x)$ may be found that $\|P(x)-f(x)\|<\varepsilon$.

As a result of the problem solution, the points $A\left(X_{A}, Y_{A}, Z_{A}\right)$ are determined in the fixed system of coordinates, $B^{(0)} \in Q_{1}, C^{(0)} \in Q_{2}$, such that when coinciding the link $B C$ with them, we obtain the desired RKC in form of an open four-link chain $A B C D$.

Then we check the accuracy of the prescribed function reproduction by analysis of the position of RKC $A B C D$. If the accuracy of reproduction satisfies the prescribed function, the iteration is completed, and if it does not satisfy the prescribed accuracy, it is necessary to proceed to item 1 of the prescribed algorithm.

When specifying a part of the desired synthesis parameters in various combinations, we obtain different modifications of RKC (Kosbolov and Rakhmatulina, 2013b).

- If the coordinates of point $A\left(X_{A_{i}}, Y_{A_{i}}, Z_{A i}\right)$ and Eulerian angles $\theta_{i}^{1}, \psi_{i}^{1}, \phi_{i}^{1}$ of the solid $Q_{1}$ as well as the axes of point $D_{i}\left(X_{D_{i}}, Y_{D_{i}}, Z_{D i}\right)$ and Eulerian angles $\theta_{i}^{1}, \psi_{i}^{1}, \phi_{i}^{1}$ of the solid $Q_{2}$ are specified, we obtain a three-link open chain $A B C D$ (Fig. 1). The necessary conditions for the minimum of the sum $S$ in this case takes the form

$$
\frac{\partial S}{\partial j}=0 \quad j=x_{B}, y_{B}, z_{B}, R, x_{C}, y_{C}, z_{C}
$$

and to find the minimum $S$, we may use the algorithm given above, considering that the parameters $X_{A}, Y_{A}, Z_{A}$ are specified.

If the points $A\left(X_{A}, Y_{A}, Z_{A}\right)$ and $D\left(X_{D}, Y_{D}, Z_{D}\right)$ are fixed, then, as a result of the synthesis of RKC, we obtain a spatial four-link chain $A B C D$.

- Given the coordinates $x_{C}=y_{C}=z_{C}=0$ of the point $C \in Q_{2}$, coordinates $X_{D i}, Y_{D i}$, $Z_{D i}$ of the point $D$ of the solid $Q_{2}$ and Eulerian angles $\theta_{i}^{1}, \psi_{i}^{1}, \phi_{i}^{1}$ of the solid $Q_{1}$, and the desired parameters $X_{A}, Y_{A}, Z_{A}, \mathrm{R}, x_{B}, y_{B}, z_{B}$.

The necessary conditions for the minimum of the sum $S$ takes the form

$$
\frac{\partial S}{\partial j}=0 \quad j=X_{A}, Y_{A}, Z_{A}, R, x_{B}, y_{B}, z_{B}
$$

To find the minimum of the function $S$ we may use again the algorithm given above, considering that $x_{C}=y_{C}=z_{C}=0$.

- Given coordinates $x_{B}, y_{B}, z_{B}=0$ of the point $B$ of the solid $Q_{1}$ and Eulerian angles of the solid $Q_{2}, \theta_{i}^{2}, \psi_{i}^{2}, \phi_{i}^{2}$. The original problem reduces to the definition of sphere of positions of the fixed point $C$ of the solid $Q_{2}$ which is the least remote from $N$ (Fig. 1). 
The necessary conditions for the minimum of the sum $S$ is

$$
\frac{\partial S}{\partial j}=0 \quad j=X_{A}, Y_{A}, Z_{A}, R, x_{C}, y_{C}, z_{C}
$$

This problem was studied in detail in work by Kosbolov et al. (2013c). For its solution we may also use the algorithm given above, assuming $x_{B}, y_{B}, z_{B}=0$, but in this special case, the algorithm of the minimum search is absolutely coinciding with the kinematic inversion method.

Thus, as we see, the problem of RKC with spherical kinematic pairs is solved, and their modifications may be used as modules of structural and kinematic synthesis of spatial linkage mechanisms through specified positions of the input and output links.

\section{Example}

Suppose that it is necessary to design a six-linkage mechanism with spherical pairs (Fig. 2), approximately reproducing seven body positions specified in Table 1 and the initial data in Table 2.
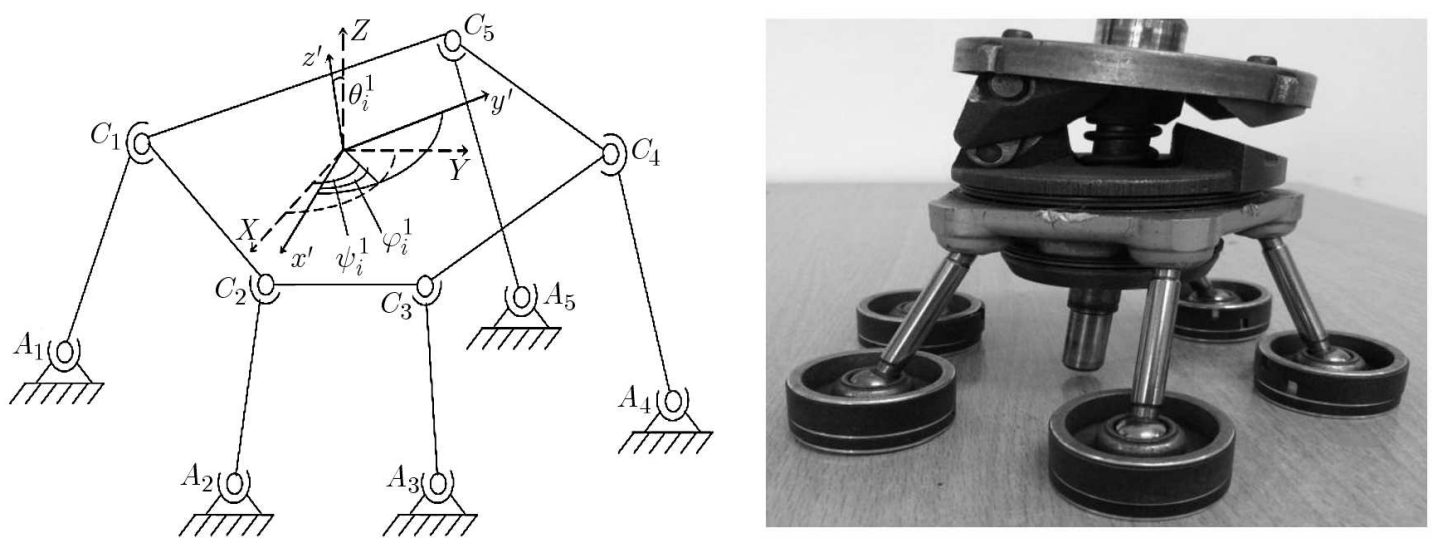

Fig. 2. Kinematic diagram of spatial linkage mechanisms with spherical pairs

Table 1. Assigned positions of the body for synthesis of a single movable mechanism - six-linkage mechanism with $N=7$

\begin{tabular}{|c|c|c|c|c|c|c|}
\hline Position & \multirow{2}{*}{$X_{O i}$} & \multirow{2}{*}{$Y_{O i}$} & \multirow{2}{*}{$Z_{O i}$} & \multicolumn{3}{|c|}{ Euler angles [deg] } \\
\cline { 5 - 7 } & & & $\theta_{i}^{1}$ & $\psi_{i}^{\perp}$ & $\phi_{i}^{\perp}$ \\
\hline \hline 1 & 0.30 & 0.12 & 0.01 & 0 & 0 & 0 \\
\hline 2 & 0.35 & 0.17 & 0.24 & 28 & 35 & 17 \\
\hline 3 & 0.44 & 0.21 & 0.25 & 34 & 38 & 5 \\
\hline 4 & 0.51 & 0.15 & 0.32 & 17 & 24 & 12 \\
\hline 5 & 0.50 & 0.30 & 0.45 & 50 & 50 & 21 \\
\hline 6 & 0.60 & 0.25 & 0.41 & 45 & 33 & 24 \\
\hline 7 & 0.55 & 0.32 & 0.35 & 0 & 0 & 0 \\
\hline
\end{tabular}

When $N=7$, as known in mobile spatial systems, there are points (not more than 20) with seven positions on one sphere. Furthermore, the points which are in the seven considered positions are approaching the sphere. As noted, the exact spherical points of a movable system correspond to an absolute minimum $S=0$ of the sum $S=\sum \Delta_{q_{i}}^{2}$, because they make all $\Delta_{q_{i}}$ 
Table 2. Initial data $(N=7)$

\begin{tabular}{|c|c|c|c|c|c|}
\hline \multirow{2}{*}{$N=7$} & \multicolumn{2}{|c|}{$\begin{array}{l}\text { ZLL }=6, \mathrm{ZKK}=6, \\
\mathrm{ZII}=6, \mathrm{dmj}=0.2\end{array}$} & \multicolumn{3}{c|}{$\mathrm{MJ}=-1.2, \mathrm{NJ} 0=-1.2}$, \\
& $\mid \mathrm{KJ} 0=-1.2$ \\
\hline \hline $\mathrm{XS}=0.3$ & $\mathrm{YS}=0.12$ & $\mathrm{ZS}=0.01$ & $\mathrm{~F}=0$ & $\mathrm{P}=0$ & $\mathrm{~T}=0$ \\
\hline $\mathrm{XS}=0.35$ & $\mathrm{YS}=0.17$ & $\mathrm{ZS}=0.24$ & $\mathrm{~F}=28$ & $\mathrm{P}=35$ & $\mathrm{~T}=17$ \\
\hline $\mathrm{XS}=0.44$ & $\mathrm{YS}=0.21$ & $\mathrm{ZS}=0.25$ & $\mathrm{~F}=34$ & $\mathrm{P}=38$ & $\mathrm{~T}=5$ \\
\hline $\mathrm{XS}=0.51$ & $\mathrm{YS}=0.15$ & $\mathrm{ZS}=0.32$ & $\mathrm{~F}=17$ & $\mathrm{P}=24$ & $\mathrm{~T}=12$ \\
\hline $\mathrm{XS}=0.5$ & $\mathrm{YS}=0.3$ & $\mathrm{ZS}=0.45$ & $\mathrm{~F}=50$ & $\mathrm{P}=50$ & $\mathrm{~T}=21$ \\
\hline $\mathrm{XS}=0.6$ & $\mathrm{YS}=0.25$ & $\mathrm{ZS}=0.41$ & $\mathrm{~F}=45$ & $\mathrm{P}=33$ & $\mathrm{~T}=24$ \\
\hline $\mathrm{XS}=0.55$ & $\mathrm{YS}=0.32$ & $\mathrm{ZS}=0.35$ & $\mathrm{~F}=0$ & $\mathrm{P}=0$ & $\mathrm{~T}=0$ \\
\hline
\end{tabular}

Table 3. Results of calculation $(N=4)$

\begin{tabular}{|c|c|c|c|}
\hline \multicolumn{2}{|c|}{$\mathrm{LL}=3, \mathrm{KK}=2, \mathrm{II}=1$} & \multicolumn{2}{|c|}{$\mathrm{MJ}=-0.6, \mathrm{NJ}=-0.8, \mathrm{KJ}=-1$} \\
\hline \hline$N=1$ & $A=0.488270$ & $C=-2.22103$ & \\
\hline$N=2$ & $A=0.303894$ & $C=3.96393$ & \\
\hline$N=3$ & $A=0.151985$ & $C=-3.85753$ & \\
\hline$N=4$ & $A=0.146845$ & $C=3.00599$ & \\
\hline$R 0=0.245293$ & $R 01=0.245194$ & $S=4.296551 \mathrm{E}-0.3$ & $S 1=4.295576 \mathrm{E}-0.3$ \\
\hline
\end{tabular}

\begin{tabular}{|c|c|c|c|}
\hline \multicolumn{2}{|c|}{$\mathrm{LL}=3, \mathrm{KK}=2, \mathrm{II}=2$} & \multicolumn{2}{c|}{$\mathrm{MJ}=-0.6, \mathrm{NJ}=-0.8, \mathrm{KJ}=-0.8$} \\
\hline \hline$N=1$ & $A=0.488270$ & $C=-3.42355$ & \\
\hline$N=2$ & $A=0.303894$ & $C=2.28575$ & \\
\hline$N=3$ & $A=0.151985$ & $C=-2.95424$ & \\
\hline$N=4$ & $A=0.146845$ & $C=3.00600$ & \\
\hline$R 0=0.245216$ & $R 01=0.245194$ & $S=4.292136 \mathrm{E}-0.3$ & $S 1=4.295576 \mathrm{E}-0.3$ \\
\hline
\end{tabular}

\begin{tabular}{|c|c|c|c|}
\hline \multicolumn{2}{|c|}{$\mathrm{LL}=3, \mathrm{KK}=2, \mathrm{II}=3$} & \multicolumn{2}{|c|}{$\mathrm{MJ}=-0.6, \mathrm{NJ}=-0.8, \mathrm{KJ}=-0.6$} \\
\hline \hline$N=1$ & $A=0.488174$ & $C=-3.18222$ & \\
\hline$N=2$ & $A=0.303906$ & $C=5.18244$ & \\
\hline$N=3$ & $A=0.152115$ & $C=-2.55608$ & \\
\hline$N=4$ & $A=-0.146845$ & $C=0.3006$ & \\
\hline$R 0=0.245195$ & $R 01=0.245194$ & $S=4.294402 \mathrm{E}-0.3$ & $S 1=4.295576 \mathrm{E}-0.3$ \\
\hline
\end{tabular}

\begin{tabular}{|c|c|c|c|}
\hline \multicolumn{2}{|c|}{$\mathrm{LL}=3, \mathrm{KK}=2, \mathrm{II}=4$} & \multicolumn{2}{|c|}{$\mathrm{MJ}=-0.6, \mathrm{NJ}=-0.8, \mathrm{KJ}=-0.6$} \\
\hline \hline$N=1$ & $A=0.488217$ & $C=-2.74528$ & \\
\hline$N=2$ & $A=0.303942$ & $C=8.77332$ & \\
\hline$N=3$ & $A=0.152078$ & $C=-2.92302$ & \\
\hline$N=4$ & $A=0.146866$ & $C=3.00599$ & \\
\hline$R 0=0.245217$ & $R 01=0.245194$ & $S=4.297097 \mathrm{E}-0.3$ & $S 1=4.295576 \mathrm{E}-0.3$ \\
\hline
\end{tabular}

$(i=1,2, \ldots, 7)$ vanish. The approximate spherical points of a movable system correspond to the local minima of the sum or are located in the vicinity of the local minimum of the function $S=F\left(x_{C}, y_{C}, z_{C}\right)$. Both these and other are common points of the surfaces $G_{x}=0, G_{y}=0$, $G_{z}=0$, and they are determined based on the solution to system (2.21).

We are definitely interested not in all system solutions (2.24), but in those which correspond to the minimum of the sum $S$. These solutions in this case are a numerical method for searching of the minimum of the sum $S$, based on the searching algorithm for the minima of the function $S$. Since we need five spherical points of the movable system for construction of the six-linkage mechanism (Fig. 2) having analysed the results we selected five points of the minimum of the 
surface $S=F\left(x_{C}, y_{C}, z_{C}\right)$. The coordinates of these points, the parameters of approaching spheres corresponding to them and the values $\Delta_{q_{i}}$ maximum per module are given in Table 3 .

\section{Research results}

Judging from deviations, the points $C_{1}, C_{2}, C_{3}$ are the exact spherical points (found with the given measure of inaccuracy), and $C_{4}$ and $C_{5}$ - are approximate points.

To solve this problem, a Visual Basic program has been written. The results of the problem solution are given in Table 3 .

The calculations have been made within the range

$$
-1.2<\mathrm{MJ}<0.6 \quad-1.2<\mathrm{NJ}<0.6 \quad-1.2<\mathrm{KJ}<0.6
$$

with a pitch $d m_{j}=0.2$.

In the entire range of calculations, the process of calculation is concurrent. The global minimum is equal to

$$
S_{\text {min }}=0.0042921
$$

and it is achieved at

$$
\mathrm{LL}=3 \quad \mathrm{KK}=2 \quad \mathrm{II}=2
$$

In Table 3, only a part of results in the neighborhood of the global minimum is shown. Its value is highlighted in bold frame in Table 3 .

Below, the results of calculation of the objective function in form of carpet plots and 3D plots (Figs. 3 and 4) are given.
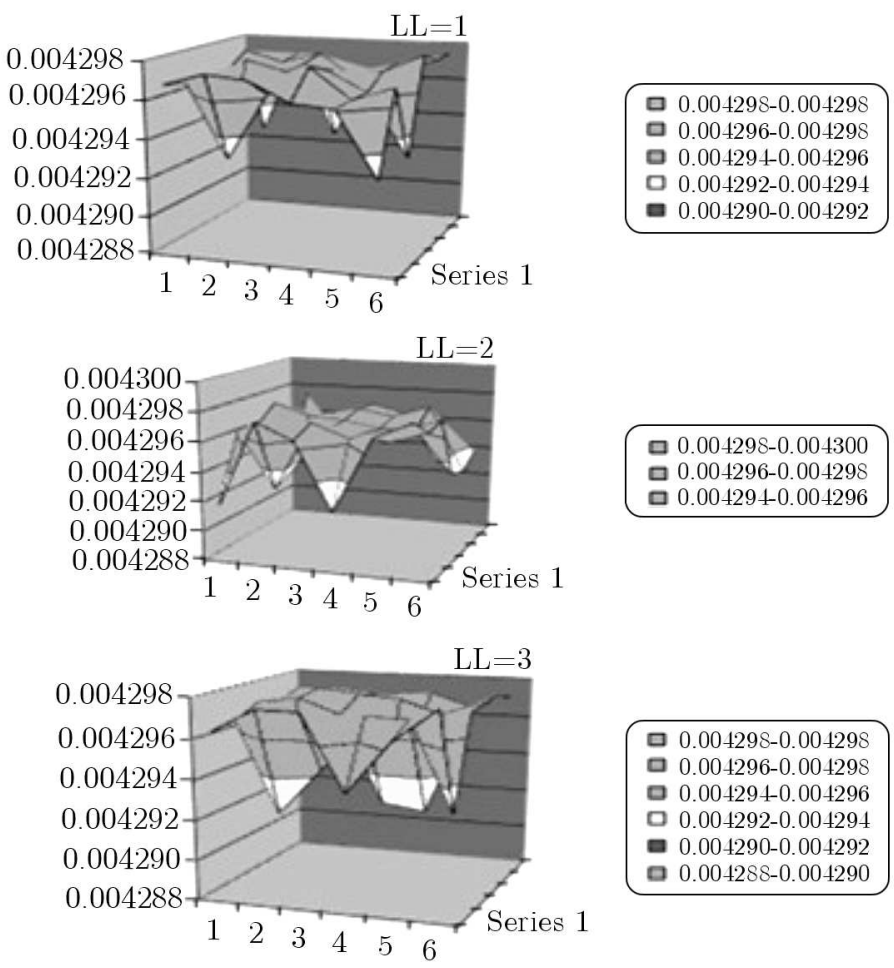

Fig. 3. $\mathrm{LL}=1,2,3, S_{\min }=-0.0042921$, volume graphics 

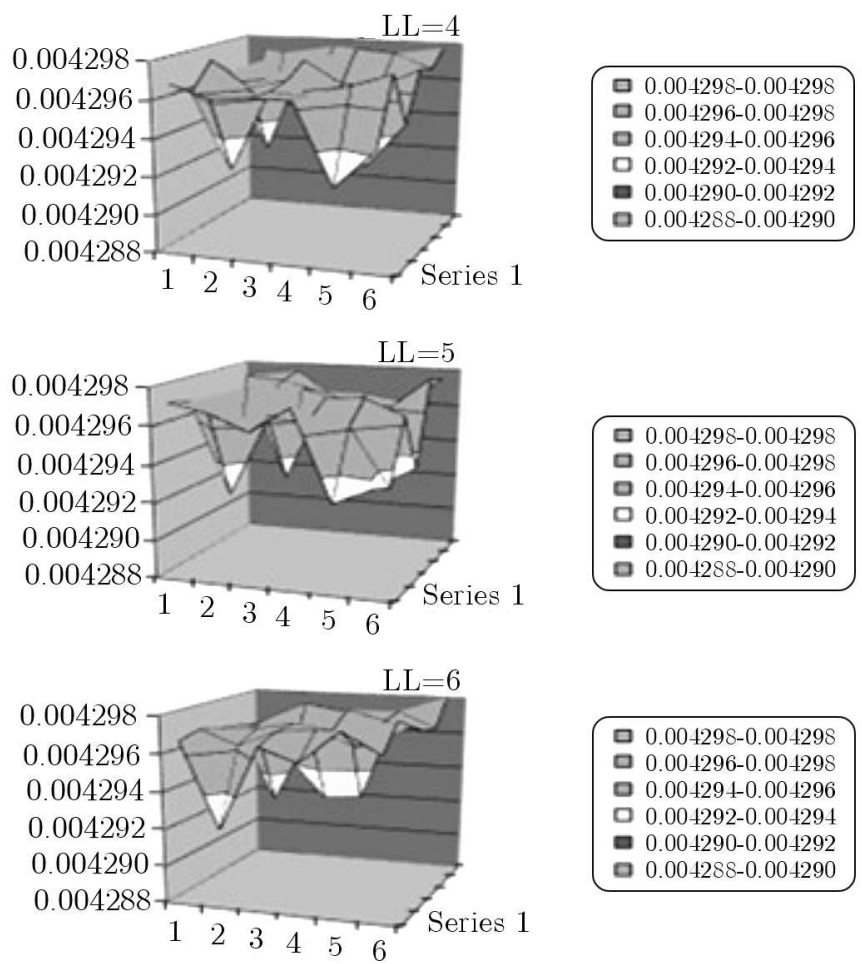

Fig. 4. $\mathrm{LL}=4.5,6, S_{\min }=-0.0042921$, volume graphics

\section{Discussion}

As can be concluded, the use of one and the same objective function being generated for synthesis of $\mathrm{BKC}$ and its modification allows automating of the process of synthesis of spatial linkage mechanisms through predetermined positions of the input and output links of the mechanism.

\section{Conclusions}

In summary, in the synthesis of BKC with spherical kinematic pairs through predetermined positions of the input and output links of a mechanism when two adjacent links of BKC tend to infinity, it is necessary to replace the spherical kinematic pair for a plain or cylindrical one. In such a case, the synthesized mechanism takes form of a spatial link mechanism after determining the required parameters.

\section{References}

1. Golynski Z., 1970, Optimal synthesis problems solved by means of nonlinear programming and random methods, Journal of Mechanisms, 3, 287-309

2. InNocenti C., 1995, Direct kinematics in analytical form of the 6-4 fully - parallel mechanisms, Transactions of the ASME, Journal of Mechanical Design, 1, 85-95

3. Joldasbekov W.A., Drakunov Y.M., Kosbolov S.B., Moldabekov M.M., 1987, Synthesis of starting kinematics mechanisms. High grade Math., Academy of Sciences of the Kazakh SSR, series Science, 3, 65-70

4. Kosbolov S.B., BeKenov E.T., 2013, Kinematic synthesis of spatial six-membered transfer mechanism based on the original kinematic chains, Bulletin of Science and Technological, 6, 30-38 
5. Kosbolov S., Moldabekov M., Bekenov E., 2005, Kinematic synthesis of spatial lever motion - generating mechanisms by use of initial kinematic chain, The 9-th IFToMM, International Symposium on Theory of Machines and Mechanisms, 4, 245-250

6. Kosbolov S.B., Rakhmatulina A.B., 2012a, Optimization flow of force of plane leverage mechanisms, International Conference on European Science and Technology, 271-276

7. Kosbolov S.B., Rakhmatullina A.B., 2012b, Parametric synthesis of spatial linkage based on the initial kinematic chain, Modern Problems of Science and Education, 2, 207-215

8. Kosbolov S.B., Rakhmatulina A.B., 2013a, Design of a new load lifting mechanism, Procedia Social and Behavioral Sciences, 83, 689-692

9. Kosbolov S.B., Rakhmatulina A.B., 2013b, Kinematic synthesis of three-dimensional six-link motion-generating mechanisms in the basis of initial kinematic chains, Journal of Machinery Manufacture and Reliability, 2, 102-108

10. Kosbolov S.B., Rakhmatulina A.B., 2013c, Synthesis mechanism to compensate weft threads on a multicolored weaving loom machine shuttleless, Tekstil ve Konfeksiyon, Turkey, 23, 23-31

11. Kosbolov S.B., Rakhmatulina A.B., Imanbaeva N.C., Zhaurt A., 2014, Optimal design of leading kinematical chain of eight linked planar load lifting linkage, Mediterranean Journal of Social Sciences, 23, 2656-2660

12. MCCARThy J.M., 1995, The synthesis of planar RR and spatial CC chains and the equation of a triangle, Transactions of the ASME, Journal of Mechanical Design, 101-106

13. Vorobyov E.I., Egorov O.D., Popov S.A., 1988, Mechanics of industrial robots, Calculation and Design of Mechanisms, 366-376 